\title{
Interferon therapy reduces the risk for hepatocellular carcinoma
}

Yoshida $\mathrm{H}$, Shiratori $\mathrm{Y}$, Moriyama $\mathrm{M}$, et al. Interferon therapy reduces the risk for hepatocellular carcinoma: national surveillance program of cirrhotic and noncirrhotic patients with chronic hepatitis C in Japan. Ann Intern Med 1999;131:174-81.

\section{Object}

To examine the effect of interferon on the risk of hepatitis C infected individuals developing hepatocellular carcinoma after adjusting for other risk factors including the degree of fibrosis.

\section{Methods}

Multicentre retrospective cohort study of 2890 Japanese patients with hepatitis C, of whom 2400 received interferon and 490 did not. All cases had undergone liver biopsy within a year of commencement of treatment and minimum follow up was one year (including six monthly ultrasound examinations). Risk factors examined included age, degree of fibrosis, transaminase levels, viral load and genotype. Analysis was by intention to treat using Cox proportional hazards regression analysis.

\section{Results}

The untreated patients had similar clinical and demographic characteristics except that a higher proportion of untreated cases had cirrhosis (stage F4) at diagnosis, but fewer had stage F3 compared with the treated cohort and viral load was higher in the untreated group, although transaminase levels were lower. During a median follow up of 4.3 years there were $89 / 2400$ hepatocellular carcinomas in the treated group compared with $59 / 490$ in those not treated with interferon, giving a highly significant risk reduction after adjustment for degree of fibrosis and other factors (adjusted risk ratio 0.516, 95\% CI 0.358, $0.742, \mathrm{p}<0.001)$. Other independent factors associated with a risk reduction were normal or minimally raised (less than twice normal) levels of transaminases and a sustained virological response.

\section{Conclusion}

In this large cohort study of almost 3000 cases, interferon treatment was an independent factor associated with a $50 \%$ reduction in the risk of developing hepatocellular carcinoma, regardless of stage of fibrosis or the presence of cirrhosis, especially for individuals with sustained biochemical or virological response.

\section{Comment}

The uncertainties in the natural history of hepatitis $\mathrm{C}$ virus (HCV) infection and the inadequacies of current antiviral therapies continue to challenge those with clinical and financial responsibility for the care of patients with this apparently indolent disease. Nevertheless, it is clear that most patients infected with HCV develop chronic infection and that a proportion of these, in time, will develop cirrhosis and that a proportion of these, in time, will develop hepatocellular carcinoma (HCC). The proportions and time intervals remain unclear and variables such as age at exposure, mode of infection, race, sex, alcohol consumption, and genotype of virus are frequently cited as cofactors that modify disease progression.

The traditional goals for antiviral therapy comprise sustained clearance of viral nucleic acid from the serum and liver with amelioration of biochemical and histopathological necroinflammatory markers. The conventional rationale for antiviral therapy has been to achieve these goals before the development of advanced fibrotic liver disease. Although we have acquired enormous global experience in the treatment of precirrhotic liver disease in only a decade, relatively few studies of long term benefit to patients with advanced liver disease have been reported. This is because: (i) the traditional therapeutic goals are harder to achieve; (ii) the conventional rationale is irreversibly inapplicable; (iii) few centres have enough untreated patients with advanced liver disease to evaluate in a randomised prospective manner; (iv) the required length of follow up is approaching the duration that effective antiviral therapy has been available to humans; and (v) the availability of liver transplantation raises ethical issues in study design.

For these reasons, nearly all of our data concerning the effects of interferon on the development of HCC in patients with HCV cirrhosis have been derived from multicentre retrospective cohort studies with the control group comprised of patients that did not want to have therapy or their doctors believed treatment to be contraindicated. Global applicability of these data is confounded by the observation that the duration of infection may differ by several decades in different populations, "apparently" at the same stage of disease.

Only two randomised prospective studies appear to have been undertaken. In the first, ${ }^{1} 45 \mathrm{HCV}$ cirrhotic patients were randomised to receive interferon therapy and 45 were followed as controls. A sustained antiviral response was seen in seven of the treated patients but HCC developed in only two patients during follow up ranging from two to seven years. Seventeen HCCs developed in the control group, suggesting that interferon has a protective effect, independent of the requirement for a sustained virological response. In the second trial, ${ }^{2} 47 \mathrm{HCV}$ cirrhotic patients received interferon and 39 were followed as controls. Only two patients had a sustained response to treatment and five HCCs developed in three years of follow up. The control group developed nine HCCs during this time, a nonsignificant result.

Of the retrospective cohort studies, the paper of Yoshida et al is noteworthy because the effect of interferon on HCC development was assessed in 2400 patients at all stages of liver disease with stratification at four levels of fibrosis. Viral load, genotype, and alcohol consumption were included in the analysis, and follow up was for a median of 4.3 years. Overall, HCC developed in $12 \%$ of controls and $3.7 \%$ of treated patients $(1.2 \%$ in the responders and $4.8 \%$ in the non-responders). The annual incidence of HCC was 
$7.9 \%$ in cirrhotics but the effects of interferon did not reach statistical significance in this subgroup. The likely explanation is that there were insufficient sustained responders because highly significant results were obtained in the precirrhotic fibrotic groups with a lower annual incidence of HCC but a greater proportion of sustained responders. Viral load and genotype were not risk factors for HCC although a low viral load and genotype 2 predisposed to a sustained antiviral response. One other study ${ }^{3}$ has examined the effects of interferon on the incidence of HCC at all stages of fibrosis, but this was unstratified. The risk ratios for the development of HCC for patients with a sustained response, relapsed response, and no response, compared with controls, were $0.06,0.51$, and 0.95 , respectively.

Four other retrospective case control cohort studies have been restricted to patients with cirrhosis. In three, ${ }^{4-6}$ sustained responders had a significant reduction in the incidence of HCC, and treated patients without a sustained response demonstrated intermediate benefit. Advanced age, male sex, and features of portal hypertension were risk factors for HCC development. In the fourth, ${ }^{7}$ there was no difference in survival between cases and controls (91\% at five years and $79 \%$ at 10 years), highlighting the slowly progressive nature of chronic liver disease in some populations. ${ }^{8}$ In a descriptive study (no untreated control group), ${ }^{9}$ the seven year cumulative incidences of HCC were $4.3 \%, 4.7 \%$, and $26.1 \%$ in sustained, transient, and non-responders, respectively. Again, advanced age and male sex were risk factors for HCC, as they were in the most recent European publication ${ }^{10}$ documenting a five year incidence rate for HCC of $13.4 \%$.

Where does this leave us? It would appear that elderly males with features of portal hypertension are the most likely to develop HCC in current practice. Sustained termination of viral replication will significantly reduce their chances of developing HCC although current therapies are unlikely to achieve this. We can only speculate on the protective efficacy of combination antiviral therapy as the duration of follow up is still too short to be meaningful. There are data to suggest that interferon therapy may benefit patients independently of a sustained virological response and further randomised prospective studies would be reassuring to confirm this in different populations.

Cranfield University Postgraduate Medical School in Gloucester, J L BROWN Gloucestershire Royal Hospital, Gloucester GL1 3NN, UK

j.brown@rmcs.cranfield.ac.uk

1 Nishiguchi S, Kuroki T, Nakatani S, et al. Randomised trial of effects of interferon- $\alpha$ on incidence of hepatocellular carcinoma in chronic active hepatitis C with cirrhosis. Lancet 1995;346:1051-5.

2 Valla D-C, Chevallier M, Marcellin P, et al. Treatment of hepatitis C virusrelated cirrhosis: A randomized, controlled trial of interferon alfa- $2 \mathrm{~b}$ versus no treatment. Hepatology 1999;29:1870-5.

3 Imai Y, Kawata S, Tamura S, et al. Relation of interferon therapy and hepatocellular carcinoma in patients with chronic hepatitis C. Ann Intern Med 1998;129:94-9.

4 International Interferon- $\alpha$ Hepatocellular Carcinoma Study Group. Effect of interferon- $\alpha$ on progression of cirrhosis to hepatocellular carcinoma: a retrospective cohort study. Lancet 1998;351:1535-9.

5 Serfaty L, Aumaitre H, Chazouillieres O, et al. Determinants of outcome of compensated hepatitis C virus-related cirrhosis. Hepatology 1998;27:143540

6 Mazzella G, Accogli E, Sottili S, et al. Alpha interferon treatment may prevent hepatocellular carcinoma in HCV-related liver cirrhosis. 7 Hepatol 1996;24:141-7.

7 Fattovich G, Giustina G, Degos F, et al. Morbidity and mortality in compensated cirrhosis type C: A retrospective follow-up study of 384 patients. Gastroenterology 1997;112:463-72.

8 Seeff L, Miller R, Rabkin C, et al. 45-year follow-up of hepatitis C virus infection in healthy young adults. Ann Intern Med 2000;132:105-11.

9 Kasahara A, Hayashi N, Mochizuki K, et al. Risk factors for hepatocellular carcinoma and its incidence after interferon treatment in patients with chronic hepatitis C. Hepatology 1998;27:1394-402.

10 Degos F, Christidis C, Ganne-Carrie N, et al. Hepatitis C virus related cirrhosis: time to occurrence of hepatocellular carcinoma and death. Gut 2000;47:131-6. 